\title{
What Is the Difference between Gamma and Gaussian Distributions?
}

\author{
Xiao-Li Hu \\ School of Electrical Engineering and Computer Science, University of Newcastle, Newcastle, Australia \\ Email: xiaoli.hu@newcsatle.edu.au, xlhu@amss.ac.cn
}

Received November 19, 2012; revised December 25, 2012; accepted January 3, 2013

\begin{abstract}
An inequality describing the difference between Gamma and Gaussian distributions is derived. The asymptotic bound is much better than by existing uniform bound from Berry-Esseen inequality.
\end{abstract}

Keywords: Gamma Distribution; Gaussian Distribution; Berry-Esseen Inequality; Characteristic Function

\section{Introduction}

\subsection{Problem}

We first introduce some notations. Denote Gamma distribution function as

$$
\Gamma(k, x)=\frac{1}{\Gamma(k)} \int_{0}^{x} t^{k-1} \mathrm{e}^{-t} \mathrm{~d} t
$$

for $k>0$ and $x \geq 0$, where $\Gamma(k)$ is the Gamma function, i.e.,

$$
\Gamma(k)=\int_{0}^{\infty} t^{k-1} \mathrm{e}^{-t} \mathrm{~d} t .
$$

Assume $\Gamma(k, x)=0$ for $x<0$. The density of chisquare distributed random variable $\chi_{n}$ with $n$ degrees of freedom is

$$
f_{\chi}(x, n)=\left\{\begin{array}{lr}
\frac{1}{2^{\frac{n}{2}} \Gamma\left(\frac{n}{2}\right)} x^{\frac{n}{2}-1} \mathrm{e}^{-\frac{x}{2}}, & \text { for } x>0, \\
0, & \text { otherwise. }
\end{array}\right.
$$

It is well-known that the random variable $\chi_{n}$ can be interpreted by $\chi_{n}=\sum_{k=1}^{n} \eta_{k}^{2}$ with $n$ independent and identically distributed (i.i.d.) random variables $\eta_{k} \sim \mathcal{N}(0,1)$, $k=1,2, \cdots, n$ where $\mathcal{N}(0,1)$ denotes the standard Gaussian distribution. The mean and variance of $\chi_{n}$ is respectively

$$
E \chi_{n}=n, E\left(\chi_{n}-n\right)^{2}=2 n .
$$

Then, by simple change of variable we find

$$
P\left[\frac{\chi_{n}-n}{\sqrt{2 n}}<x\right]=\Gamma\left(\frac{n}{2}, \frac{n}{2}+x \sqrt{\frac{n}{2}}\right) .
$$

On the other side, by the Berry-Esseen inequality to $\left\{\left(\eta_{k}^{2}-1\right) / \sqrt{2}, k=1, \cdots, n\right\}$, it is easy to find a bound $C>0$ such that

$$
\left|P\left[\frac{\chi_{n}-n}{\sqrt{2 n}}<x\right]-\Phi(x)\right| \leq \frac{C}{\sqrt{n}},
$$

where $\Phi(x)$ is the standard Gaussian distribution function, i.e.,

$$
\Phi(x)=\int_{-\infty}^{x} \frac{1}{\sqrt{2 \pi}} \mathrm{e}^{-\frac{x^{2}}{2}} \mathrm{~d} x .
$$

Then, by Equations (2) and (3) it follows

$$
\left|\Gamma\left(\frac{n}{2}, \frac{n}{2}+x \sqrt{\frac{n}{2}}\right)-\Phi(x)\right| \leq \frac{C}{\sqrt{n}},
$$

which describes the distance between Gamma and Gaussian distributions. The purpose of this paper is to derive asymptotic sharper bound $C$ in Equation (5), which much improves the constant $C$ by directly using Berry-Esseen inequality. The main framework of analysis is based on Gil-Pelaez formula (essentially equivalent to Levy inversion formula), which represents distribution function of a random variable by its characteristic function.

The main result of this paper is as following.

Theorem 1.1 A relation of the Gamma distribution (1) and Gaussian distribution (4) is given by

$$
\sup _{x}\left|\Gamma\left(\frac{n}{2}, \frac{n}{2}+x \sqrt{\frac{n}{2}}\right)-\Phi(x)\right| \leq \frac{C(n)}{\sqrt{n}},
$$

where

$$
C(n)=\frac{1}{3 \sqrt{\pi}}+C_{1}(n),
$$




$$
\begin{aligned}
C_{1}(n) & =\frac{1}{3 \sqrt{\pi} n^{\delta}}+\frac{3}{2 \sqrt{2 \pi n}} \\
& +\frac{\sqrt{n} \mathrm{e}^{-\frac{n_{0}^{2}}{2}}}{\pi n_{0}^{2}}+\frac{\sqrt{n}\left(n_{1}-n_{0}\right)}{\pi n_{0} \mathrm{e}^{n_{0}^{2} / 6}}+\frac{2}{\sqrt{n} \pi}
\end{aligned}
$$

with $n_{1}=\sqrt{\frac{n}{2}}$ and $n_{0}=n^{\frac{1}{6}-\frac{\delta}{3}}$ for any $\delta \in\left(0, \frac{1}{2}\right)$.

Clearly, $C_{1}(n) \rightarrow 0$ as $n \rightarrow \infty$. Thus, the asymptotical bound is

$$
C(n) \rightarrow \frac{1}{3 \sqrt{\pi}}=0.1881 \cdots
$$

as $n \rightarrow \infty$. To check the tightness of the limit value of $C(n)$, we plot in Figure 1 the multiplication

$$
\sqrt{n} \cdot \sup _{-\sqrt{\frac{n}{2}} \leq x \leq \sqrt{\frac{n}{2}}}\left|\Gamma\left(\frac{n}{2}, \frac{n}{2}+x \sqrt{\frac{n}{2}}\right)-\Phi(x)\right|
$$

for $n=1,2, \cdots, 200$, where the straight line is the limit value $\frac{1}{3 \sqrt{\pi}}$. From this experiment it seems that $\frac{1}{3 \sqrt{\pi}}$ is the best constant. The tendency of the theoretical formula $C(n)$ is plotted for $n \in[1,10] \times 10^{14}$ in Figure 2, which also shows the tendency to the limit value $\frac{1}{3 \sqrt{\pi}}$. The slow trend is due to that some upper bounds formulated over interval $\left[n_{0}, n_{1}\right]$ have been weakly estimated, e.g., the third and fourth terms of $C_{1}(n)$.

\subsection{Comparison to the Bound Derived by Berry-Esseen Inequality}

Let $\left\{X_{1}, X_{2}, \cdots\right\}$ be a sequence of independent identically distributed random variables with $E X_{1}=0 E X_{1}^{2}=1$

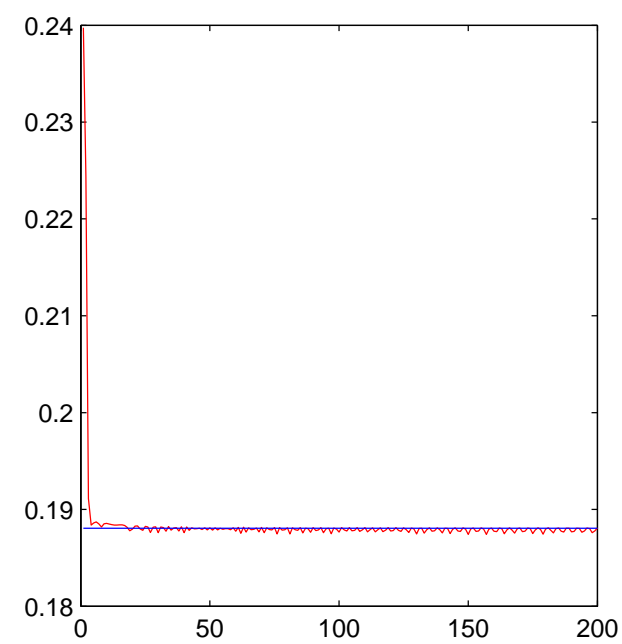

Figure 1. Experiment.

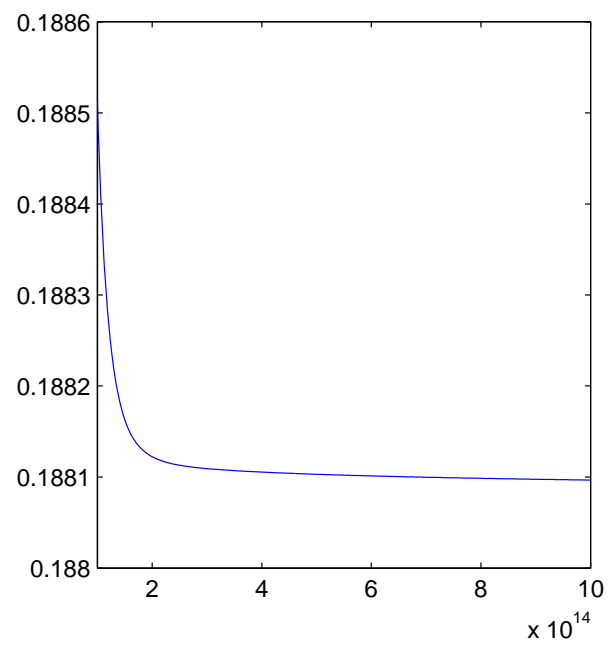

Figure 2. Trend of $C(n)$.

and finite third absolute moment $\gamma_{3}=E\left|X_{1}\right|^{3}<\infty$. Denote

$$
F_{n}(x)=P\left[\frac{X_{1}+\cdots+X_{n}}{\sqrt{n}}<x\right] .
$$

By classic Berry-Esseen inequality, there exists a finite positive number $C_{0}$ such that

$$
d\left(F_{n}, \Phi\right) \triangleq \sup _{x}\left|F_{n}(x)-\Phi(x)\right| \leq \frac{C_{0} \gamma_{3}}{\sqrt{n}} .
$$

The best upper bound $C_{0} \leq 0.4785$ is found in [1] in 2009. The bound is improved in [2] at some angle in a slight different form as

$$
d\left(F_{n}, \Phi\right) \leq \frac{C_{1}}{\sqrt{n}}
$$

with

$$
C_{1}=\min \left\{0.33477\left(\gamma_{3}+0.429\right), 0.3041\left(\gamma_{3}+1\right)\right\} .
$$

The inequality (8) will be sharper than Equation (7) for $\gamma_{3} \geq 1.93$.

Now let us derive the constant $C$ in (5) by applying Berry-Esseen inequality to $\left\{\left(\eta_{k}^{2}-1\right) / \sqrt{2}, k=1,2, \cdots\right\}$. It is difficult to calculate the exact value of third absolute moment of the random variable $\left(\eta_{1}^{2}-1\right) / \sqrt{2}$. Thus, it is approximated as

$$
\gamma_{3}=\frac{E\left|\eta_{1}^{2}-1\right|^{3}}{2 \sqrt{2}}=3.0731 \cdots
$$

by using Matlab to integrate over interval $[0,100]$ divided equivalently 100,000 subinterval for its half value.

By Equation (7) with $C_{0}=0.4785$ we have

$$
C_{0} \gamma_{3}=1.4705 \cdots
$$

and by Equation (8) we have 


$$
C_{1}=1.1724 \cdots .
$$

Hence, the best constant $C$ in Equation (5) by applying Berry-Esseen inequality is $1.1724 \cdots$. Obviously, the limit bound

$$
\lim _{n \rightarrow \infty} C(n)=\frac{1}{3 \sqrt{\pi}}=0.1881 \cdots
$$

found in this paper for chi-square distribution is much better.

The technical reason is that the Berry-Esseen inequality deals with general i.i.d. random sequences without exact information of the distribution.

\section{Proof of Main Result}

Before to prove the main result, we first list a few lemmas and introduce some facts of characteristic function theory.

\subsection{Some Lemmas}

Lemma 2.1 For a complex number $z$ satisfying $|z| \leq 1$, $\left|\mathrm{e}^{z}-1\right| \leq|z|(1+|z|)$.

Proof First show that

$$
\left|e^{z}-1-z\right| \leq|z|^{2} .
$$

By Taylor's expansion and noting $|z| \leq 1$, we have

$$
\begin{aligned}
\left|\mathrm{e}^{z}-1-z\right| & =\left|\sum_{k=2}^{\infty} \frac{z^{k}}{k !}\right| \leq \sum_{k=2}^{\infty} \frac{|z|^{k}}{k !} \\
& \leq \frac{|z|^{2}}{2} \sum_{k=0}^{\infty}\left(\frac{|z|}{2}\right)^{k} \leq|z|^{2} .
\end{aligned}
$$

Together with

$$
\left|\mathrm{e}^{z}-1\right|-|z| \leq\left|\mathrm{e}^{z}-1-z\right|,
$$

the assertion follows.

Lemma 2.2 For a real number $x$ satisfying $|x|<1$,

$$
(1+x \mathrm{i})^{\frac{1}{x \mathrm{i}}}=\exp \left(1-\frac{x \mathrm{i}}{2}+R_{3}(x)\right)
$$

where $\mathrm{i}$ is the imaginary unit and

$$
\begin{aligned}
R_{3}(x) & =-\left(\frac{x^{2}}{3}-\frac{x^{4}}{5}+\frac{x^{6}}{7}-\cdots\right) \\
& +\mathrm{i}\left(\frac{x^{3}}{4}-\frac{x^{5}}{6}+\frac{x^{7}}{8}-\cdots\right) .
\end{aligned}
$$

Clearly,

$$
\left|R_{3}(x)\right| \leq \frac{1}{3}|x|^{2}+\frac{1}{4}|x|^{3} .
$$

Proof. By Taylor expansion for complex function, for $|x|<1$ we have

$$
\begin{aligned}
& \frac{1}{x \mathrm{i}} \ln (1+x \mathrm{i})=\frac{1}{x \mathrm{i}}\left(x \mathrm{i}-\frac{(x \mathrm{i})^{2}}{2}+\frac{(x \mathrm{i})^{3}}{3}-\frac{(x \mathrm{i})^{4}}{4}+\cdots\right) \\
& =1-\frac{x \mathrm{i}}{2}+\frac{(x \mathrm{i})^{2}}{3}-\frac{(x \mathrm{i})^{3}}{4}+\cdots \\
& =1-\frac{x \mathrm{i}}{2}+R_{3}(x),
\end{aligned}
$$

where $R_{3}(x)$ is shown above. By further noting the two alternating real series above, it follows the upper bound.

We cite below a well-known inequality [3] as a lemma.

Lemma 2.3 The tail probability of the standard normal distribution satisfies

$$
\frac{x}{1+x^{2}} \cdot \frac{1}{\sqrt{2 \pi}} \mathrm{e}^{-\frac{1}{2} x^{2}}<\int_{x}^{\infty} \frac{1}{\sqrt{2 \pi}} \mathrm{e}^{-\frac{1}{2} t^{2}} \mathrm{~d} t<\frac{1}{x} \cdot \frac{1}{\sqrt{2 \pi}} \mathrm{e}^{-\frac{1}{2} x^{2}}
$$

for $x>0$.

\subsection{Characteristic Function}

Let us recall, see e.g., [4], the definition and some basic facts of characteristic function (CF), which provides another way to describe the distribution function of a random variable. The characteristic function of a random variable $X$ is defined by

$$
\varphi_{X}(t)=E \mathrm{e}^{\mathrm{i} t X},
$$

where $\mathrm{i}$ is the imaginary unit, and $t \in R$ is the argument of the function. Clearly, the $\mathrm{CF}$ for random variable $Y=a X+b$ with real numbers $a$ and $b$ is

$$
\varphi_{Y}(t)=\varphi_{X}(a t) \mathrm{e}^{\mathrm{i} b t} .
$$

Another basic quality is

$$
\varphi_{Z}(t)=\varphi_{X}(t) \varphi_{Y}(t)
$$

for $Z=X+Y$ with $X$ and $Y$ independent to each other.

It is well-known that the $\mathrm{CF}$ of standard Gaussian $\mathcal{N}(0,1)$ is

$$
\phi(t)=\mathrm{e}^{-\frac{1}{2} t^{2}}
$$

and the CF of chi-square distributed variable $\chi_{n}$ is

$$
\varphi_{\chi_{n}}(t)=(1-2 \mathrm{i} t)^{-\frac{n}{2}} \text {. }
$$

Thus, the CF for $S=\frac{\chi_{n}-n}{\sqrt{2 n}}$ is

$$
\varphi_{S}(t)=\left(1-\frac{\sqrt{2} \mathrm{i} t}{\sqrt{n}}\right)^{-\frac{n}{2}} \mathrm{e}^{-\frac{\sqrt{n i t}}{\sqrt{2}}} .
$$

The CF is actually an inverse Fourier transformation 
of density function. Therefore, distribution function can be expressed by CF directly, e.g., Levy inversion formula. We use another slightly simpler formula. For a univariate random variable $X$, if $x$ is a continuity point of its distribution $F_{X}$, then

$$
F_{X}(x)=\frac{1}{2}+\int_{0}^{\infty} \frac{\mathrm{e}^{\mathrm{i} \mathrm{i} x} \varphi_{X}(-t)-\mathrm{e}^{-\mathrm{i} \mathrm{i} x} \varphi_{X}(t)}{2 \pi \mathrm{i} t} \mathrm{~d} t,
$$

which is called Gil-Pelaez formula, see, e.g., page 168 of [4].

\subsection{Proof of Main Result}

We are now in a position to prove the main result.

Proof of Theorem 1.1 First analyze CF of $S=\frac{\chi_{n}-n}{\sqrt{2 n}}$ given by Equation (10). Denote $x=-\frac{\sqrt{2} t}{\sqrt{n}}$. For $|x|<1$, i.e., $|t|<\sqrt{\frac{n}{2}}$, by Lemma 2.2 ,

$$
\begin{aligned}
\varphi_{S}(t) & =\left(1-\frac{\sqrt{2} \mathrm{i} t}{\sqrt{n}}\right)^{\frac{-\sqrt{n}}{\sqrt{2} i t} \cdot \sqrt{n i t}} \sqrt{\sqrt{2}} \mathrm{e}^{-\frac{\sqrt{n} \mathrm{i} t}{\sqrt{2}}} \\
& =\exp \left(-\frac{t^{2}}{2}+\bar{R}_{3}(t)\right),
\end{aligned}
$$

where

$$
\begin{aligned}
\bar{R}_{3}(t) & =\frac{\sqrt{n i t}}{\sqrt{2}} R_{3}(x) \\
& =-\frac{\sqrt{n i t}}{\sqrt{2}}\left(\frac{x^{2}}{3}-\frac{x^{4}}{5}+\cdots\right) \\
& -\frac{\sqrt{n} t}{\sqrt{2}}\left(\frac{x^{3}}{4}-\frac{x^{5}}{6}+\cdots\right) .
\end{aligned}
$$

Clearly,

$$
\begin{aligned}
\left|\bar{R}_{3}(t)\right| & \leq \frac{\sqrt{n}|t|}{\sqrt{2}}\left(\frac{1}{3}|x|^{2}+\frac{1}{4}|x|^{3}\right) \\
& =\frac{\sqrt{2}|t|^{3}}{3 \sqrt{n}}+\frac{t^{4}}{2 n} .
\end{aligned}
$$

To make sure $\left|\bar{R}_{3}(t)\right|<n^{-\delta}$ for some $\delta \in(0,0.5)$, denote $n_{0}=n^{\frac{1}{6}-\frac{\delta}{3}}$. Then, it is easy to see that

$$
\begin{aligned}
\left|\bar{R}_{3}(t)\right| & \leq \frac{\sqrt{2}|t|^{3}}{3 \sqrt{n}}+\frac{t^{4}}{2 n} \\
& \leq\left(\frac{\sqrt{2}}{3}+\frac{1}{2}\right) \frac{1}{n^{\delta}}<\frac{1}{n^{\delta}} \leq 1
\end{aligned}
$$

for $|t| \leq n_{0}$. Hence, by Equations (12) and (13) and Lemma 2.1,

$$
\begin{aligned}
& \left|\varphi_{S}(t)-\phi(t)\right|=\mathrm{e}^{-\frac{t^{2}}{2}}\left|\mathrm{e}^{\bar{R}_{3}(t)}-1\right| \\
& \leq \mathrm{e}^{-\frac{t^{2}}{2}}\left|\bar{R}_{3}(t)\right|\left(1+\left|\bar{R}_{3}(t)\right|\right) \leq \mathrm{e}^{-\frac{t^{2}}{2}}\left(1+n^{-\delta}\right)\left|\bar{R}_{3}(t)\right|
\end{aligned}
$$

for $|t| \leq n_{0}$.

Now let us consider the difference between $\varphi_{S}(t)$ and $\phi(t)$, i.e., the CF (9) of Gaussian distribution, over the interval $\left[0, n_{0}\right]$. By Equation (14)

$$
\begin{aligned}
& \left|\int_{0}^{n_{0}} \frac{\mathrm{e}^{-\mathrm{itx}}\left(\varphi_{S}(t)-\phi(t)\right)}{2 \pi \mathrm{i} t} \mathrm{~d} t\right| \\
& =\int_{0}^{n_{0}} \frac{\left|\varphi_{S}(t)-\phi(t)\right|}{2 \pi t} \mathrm{~d} t \leq \frac{1+n^{-\delta}}{2 \pi} \int_{0}^{n_{0}} \mathrm{e}^{-\frac{t^{2}}{2}} \frac{\left|\bar{R}_{3}(t)\right|}{t} \mathrm{~d} t \\
& \leq \frac{1+n^{-\delta}}{2 \pi}\left[\frac{\sqrt{2}}{3 \sqrt{n}} \int_{0}^{n_{0}} t^{2} \mathrm{e}^{-\frac{t^{2}}{2}} \mathrm{~d} t+\frac{1}{2 n} \int_{0}^{n_{0}}|t|^{3} \mathrm{e}^{-\frac{t^{2}}{2}} \mathrm{~d} t\right] .
\end{aligned}
$$

Note that

$$
\int_{0}^{\infty} t^{2} \mathrm{e}^{-\frac{t^{2}}{2}} \mathrm{~d} t=\frac{\sqrt{2 \pi}}{2}, \int_{0}^{\infty}|t|^{3} \mathrm{e}^{-\frac{t^{2}}{2}} \mathrm{~d} t=\frac{3 \sqrt{2 \pi}}{2},
$$

it follows

$$
\begin{aligned}
& \left|\int_{0}^{n_{0}} \frac{\mathrm{e}^{-\mathrm{i} t x}\left(\varphi_{S}(t)-\phi(t)\right)}{2 \pi \mathrm{i} t} \mathrm{~d} t\right| \\
& \leq \frac{1+n^{-\delta}}{\sqrt{n}}\left[\frac{1}{6 \sqrt{\pi}}+\frac{3}{4 \sqrt{2 \pi n}}\right] .
\end{aligned}
$$

Similarly,

$$
\begin{aligned}
& \left|\int_{0}^{n_{0}} \frac{\mathrm{e}^{\mathrm{itx}}\left(\varphi_{S}(-t)-\phi(-t)\right)}{2 \pi \mathrm{i} t} \mathrm{~d} t\right| \\
& \leq \frac{1+n^{-\delta}}{\sqrt{n}}\left[\frac{1}{6 \sqrt{\pi}}+\frac{3}{4 \sqrt{2 \pi n}}\right] .
\end{aligned}
$$

Below let us analyze the residual integrals over the interval $\left[n_{0}, \infty\right)$. By Lemma 2.3,

$$
\begin{aligned}
& \left|\int_{n_{0}}^{\infty} \frac{\mathrm{e}^{-\mathrm{itx}} \phi(t)}{2 \pi \mathrm{i} t} \mathrm{~d} t\right| \leq \int_{n_{0}}^{\infty} \frac{\mathrm{e}^{-\frac{t^{2}}{2}}}{2 \pi t} \mathrm{~d} t \\
& \leq \frac{1}{\sqrt{2 \pi} n_{0}} \int_{n_{0}}^{\infty} \frac{\mathrm{e}^{-\frac{t^{2}}{2}}}{\sqrt{2 \pi}} \mathrm{d} t \leq \frac{\mathrm{e}^{-\frac{n_{0}^{2}}{2}}}{2 \pi n_{0}^{2}} .
\end{aligned}
$$

Similarly,

$$
\left|\int_{n_{0}}^{\infty} \frac{\mathrm{e}^{\mathrm{i} t x} \phi(-t)}{2 \pi \mathrm{i} t} \mathrm{~d} t\right| \leq \frac{\mathrm{e}^{-\frac{n_{0}^{2}}{2}}}{2 \pi n_{0}^{2}} .
$$

It is somewhat difficult to analyze the residual integral 
over $\left[n_{0}, \infty\right)$ for $\varphi_{S}(t)$. We divide it into two subintervals as following:

$$
\begin{aligned}
\left|\int_{n_{0}}^{\infty} \frac{\mathrm{e}^{-\mathrm{i} t x} \varphi_{S}(t)}{2 \pi \mathrm{i} t} \mathrm{~d} t\right| & \leq \int_{n_{0}}^{\infty} \frac{\left|1-\frac{\sqrt{2} \mathrm{i} t}{\sqrt{n}}\right|^{-\frac{n}{2}} \mathrm{~d} t}{2 \pi t} \leq \int_{n_{0}}^{\infty} \frac{\left(1+\frac{2 t^{2}}{n}\right)^{-\frac{n}{4}} \mathrm{~d} t}{2 \pi t} \\
& =\left(\int_{n_{0}}^{n_{1}}+\int_{n_{1}}^{\infty}\right) \frac{\left(1+\frac{2 t^{2}}{n}\right)^{-\frac{n}{4}} \mathrm{~d} t}{2 \pi t} \triangleq I_{1}+I_{2},
\end{aligned}
$$

where $n_{1}=\sqrt{\frac{n}{2}}$.

Observe that $\frac{1}{t}\left(1+\frac{2 t^{2}}{n}\right)^{-\frac{n}{4}}$ decreases on interval $\left[n_{0}, n_{1}\right]$ and $\left(1+\frac{1}{x}\right)^{x+1}>\mathrm{e}$ for $x>1$, we have

$$
\begin{aligned}
& 2 \pi I_{1} \leq \frac{n_{1}-n_{0}}{n_{0}\left(1+\frac{2 n_{0}^{2}}{n}\right)^{\frac{n}{4}}} \\
& \leq \frac{n_{1}-n_{0}}{n_{0}\left(1+\frac{2 n_{0}^{2}}{n}\right)^{\left(\frac{n}{2 n_{0}^{2}}+1\right) \cdot \delta\left(n_{0}\right)}} \leq \frac{n_{1}-n_{0}}{n_{0} \mathrm{e}^{\delta\left(n_{0}\right)}},
\end{aligned}
$$

where

$$
\delta\left(n_{0}\right)=\frac{\frac{n}{4}}{1+\frac{n}{2 n_{0}^{2}}}=\frac{n_{0}^{2} n}{2\left(2 n_{0}^{2}+n\right)} \geq \frac{n_{0}^{2} n}{2(2 n+n)}=\frac{n_{0}^{2}}{6} .
$$

The fact $n_{0}^{2} \leq n$ is used in above formula. Thus,

$$
I_{1} \leq \frac{n_{1}-n_{0}}{2 \pi n_{0} \mathrm{e}^{n_{0}^{2} / 6}}
$$

For the other interval $\left[n_{1}, \infty\right)$, we proceed as

$$
\begin{aligned}
2 \pi I_{2} & \leq \int_{n_{1}}^{\infty} \frac{\mathrm{d} t}{t\left(1+\frac{2 t^{2}}{n}\right)^{\frac{n}{4}}} \leq \int_{n_{1}}^{\infty} \frac{\mathrm{d} t}{t\left(\frac{2 t^{2}}{n}\right)^{\frac{n}{4}}} \\
& =\left(\frac{n}{2}\right)^{\frac{n}{4}} \int_{n_{1}}^{\infty} t^{-1-\frac{n}{2}} \mathrm{~d} t=\left(\frac{n}{2}\right)^{\frac{n}{4}} \cdot \frac{2}{n}\left(\frac{n}{2}\right)^{-\frac{n}{4}}=\frac{2}{n} .
\end{aligned}
$$

By Equations (19) and (20)

$$
\left|\int_{n_{0}}^{\infty} \frac{\mathrm{e}^{-\mathrm{i} t x} \varphi_{S}(t)}{2 \pi \mathrm{i} t} \mathrm{~d} t\right| \leq \frac{n_{1}-n_{0}}{2 \pi n_{0} \mathrm{e}^{n_{0}^{2} / 6}}+\frac{1}{n \pi} .
$$

Similarly,

$$
\left|\int_{n_{0}}^{\infty} \frac{\mathrm{e}^{\mathrm{i} t x} \varphi_{S}(-t)}{2 \pi \mathrm{i} t} \mathrm{~d} t\right| \leq \frac{n_{1}-n_{0}}{2 \pi n_{0} \mathrm{e}^{n_{0}^{2} / 6}}+\frac{1}{n \pi} .
$$

By Equation (15), Equation (17), Equation (21) and Equation (16), Equation (18), Equation (22)

$$
\begin{aligned}
& \left|\int_{0}^{\infty} \frac{\mathrm{e}^{\mathrm{i} t x}\left[\varphi_{S}(-t)-\phi(-t)\right]-\mathrm{e}^{-\mathrm{i} t x}\left[\varphi_{S}(t)-\phi(t)\right]}{2 \pi \mathrm{i} t} \mathrm{~d} t\right| \\
& \leq\left|\int_{0}^{\infty} \frac{\mathrm{e}^{\mathrm{i} t x}\left[\varphi_{S}(-t)-\phi(-t)\right]}{2 \pi \mathrm{i} t} \mathrm{~d} t\right| \\
& +\left|\int_{0}^{\infty} \frac{\mathrm{e}^{-\mathrm{i} t x}\left[\varphi_{S}(t)-\phi(t)\right]}{2 \pi \mathrm{i} t} \mathrm{~d} t\right| \leq \frac{C(n)}{\sqrt{n}},
\end{aligned}
$$

where

$$
\begin{aligned}
C(n) & =\frac{1}{3 \sqrt{\pi}}+C_{1}(n), \\
C_{1}(n) & =\frac{1}{3 \sqrt{\pi} n^{\delta}}+\frac{3}{2 \sqrt{2 \pi n}} \\
& +\frac{\sqrt{n} \mathrm{e}^{-\frac{n_{0}^{2}}{2}}}{\pi n_{0}^{2}}+\frac{\sqrt{n}\left(n_{1}-n_{0}\right)}{\pi n_{0} \mathrm{e}^{n^{2} / 6}}+\frac{2}{\sqrt{n} \pi} .
\end{aligned}
$$

In view of Formula (11), the formula to be proved follows directly.

\section{REFERENCES}

[1] I. S. Tyurin, "On the Accuracy of the Gaussian Approximation," Doklady Mathematics, Vol. 80, No. 3, 2009, pp. 840-843. doi:10.1134/S1064562409060155

[2] V. Koroleva and I. Shevtsova, "An Improvement of the Berry-Essen in Equality with Application to Possion and Mixed Poison Random Sums," Scandinavian Actuarial Journal, Vol. 2012, No. 2, 2012, pp. 81-105. doi: $10.1080 / 03461238.2010 .485370$

[3] R. D. Gordon, "Values of Mills' Ratio of Area to Bounding Ordinate and of the Normal Probability Integral for Large Values of the Argument," The Annals of Mathematical Statistics, Vol. 12, No. 3, 1941, pp. 364-366. doi:10.1214/aoms/1177731721

[4] K. L. Chung, "A Course in Probability Theory," 3rd Edition, Probability and Mathematical Statistics, Academic, New York, 2001. 